\title{
Transitions in coral reef accretion rates linked to intrinsic ecological shifts on turbid-zone nearshore reefs
}

\author{
$\underline{\text { Kyle M. Morgan }}^{1}$, Chris T. Perry ${ }^{1}$, Scott G. Smithers ${ }^{2}$, Jamie A. Johnson ${ }^{1}$, and Pauline \\ Gulliver $^{3}$
}

\section{SUPPLEMENTARY INFORMATION}

\section{Radiocarbon processing and calibration methodology}

Prior to dating at the NERC Radiocarbon Facility samples selected for ${ }^{14} \mathrm{C}$ dating were sectioned, surficial calcareous encrustation removed, subjected to ultrasonic agitation in distilled water to remove detrital particles and then oven dried $\left(40^{\circ} \mathrm{C}\right)$. Samples were sent to the NERC Radiocarbon Facility-East Kilbride where the outer $20 \%$ by weight of sample was removed using dilute $\mathrm{HCl}$, rinsed in distilled water, dried, ground and converted to $\mathrm{CO}_{2}$ via hydrolysis with $85 \% \mathrm{H}_{3} \mathrm{PO}_{4}$. Sample $\mathrm{CO}_{2}$ was cryogenically purified, collected and the volume measured. $\mathrm{CO}_{2}$ was converted to graphite via Fe/Zn reduction (Sloata et al., 1987) and the ${ }^{14} \mathrm{C} /{ }^{13} \mathrm{C}$ ratio measured at the SUERC AMS Laboratory using a NEC 5MV accelerator mass spectrometer (Freeman et al., 2007) and measured to 3\%o counting precision. Results were background corrected and normalised to $\delta^{13} \mathrm{C}_{\mathrm{VPDB}} \%$ of -25 and are presented in the following Supplementary Table as: (i) conventional years Before Present (y BP) and (ii) calibrated years Before Present (calBP) where present is defined as 1950. Calibration was performed by converting the conventional dates to a calendar time-scale using the Calib 7.1 calibration program http://calib.qub.ac.uk/calib/ (Stuiver and Reimer 1993) and the Marine13 calibration curve (Reimer et al. 2013). The conventionally employed Marine Reservoir Correction in Australian waters is $450 \pm 35$ years (Gillespie, 1977). The $\Delta \mathrm{R}$ value of $+10 \pm 7$ used in Perry et al., 2012 (and references therein) was used to correct for local deviation from the Marine Reservoir Correction conventionally used for Australian waters.

Recalibration of previously reported radiocarbon results (y BP) (Palmer et al., 2010, Perry et al., 2012; 2013) was carried out using the Calib 7.1 calibration program (Stuiver and Reimer 1993) and the Marine13 calibration curve (Reimer et al., 2013) to conform with data reported here.

Process standard materials: A radiocarbon free (i.e., background) internal standard (Iceland Spar Calcite, Geo Supplies 49 Station Road, Chapeltown, Sheffield, S35 2XE) was processed contemporaneously with samples to quantify the addition of contamination during processing. All results $(n=5)$ were within $2 \sigma$ confidence limits of the of NRCF-EK hydrolysis background of $51,227 \pm 2,835$ y BP. 
Supplementary Table DR1. Summary of radiocarbon dating information from core material recovered at Offshore Paluma Shoals A (OPSA: PC1, PC5, PC6, PC7 and PC8), Offshore Paluma Shoals B (OPSB: PC1, PC3, PC4, PC5 and PC6), Offshore Paluma Shoals C (OPSC: PC1, PC2 and PC3), Offshore Paluma Shoals D (OPSD: PC1, PC2 and PC3). Sample depths are relative to lowest astronomical tide (m LAT).

\begin{tabular}{|c|c|c|c|c|c|c|c|c|}
\hline \multirow[t]{2}{*}{ Sample code } & \multirow[t]{2}{*}{ Material } & \multirow[t]{2}{*}{ Lab Ref. } & \multirow{2}{*}{$\begin{array}{l}\text { Elevation } \\
\text { (m rel. to } \\
\text { LAT) }\end{array}$} & \multirow{2}{*}{$\begin{array}{l}{ }^{14} \mathrm{C} \\
\text { age (y } \\
\mathrm{BP})\end{array}$} & \multirow{2}{*}{$\begin{array}{c}{ }^{14} \mathrm{C} \text { age } \\
\text { error (y } \\
\mathrm{BP} \text { ) }\end{array}$} & \multicolumn{2}{|c|}{$\begin{array}{c}\text { Calibrated age range } \\
(1 \sigma)\end{array}$} & \multirow{2}{*}{$\begin{array}{r}\text { Med } \\
\text { probabil } \\
\text { (cal. y }\end{array}$} \\
\hline & & & & & & Maximum & Minimum & \\
\hline \multicolumn{9}{|l|}{ OPSA } \\
\hline \multicolumn{9}{|c|}{ Core OPSA-PC1 } \\
\hline OPSA-PC1-25 & Acropora & SUERC-52297 & -1.0 & 299 & 35.7 & Modern & & Modern \\
\hline $\begin{array}{l}\text { OPSA-PC1-80 } \\
\text { OPSA-PC1- }\end{array}$ & Turbinaria & SUERC-52298 & -1.7 & 468 & 37.7 & 0 & 99 & 72 \\
\hline $\begin{array}{l}170 \\
\text { OPSA-PC1- }\end{array}$ & Platygyra & SUERC-52301 & -2.9 & 1159 & 37.7 & 659 & 726 & 697 \\
\hline 202 & Turbinaria & SUERC-52302 & -3.3 & 1211 & 37.7 & 690 & 777 & 740 \\
\hline \multicolumn{9}{|c|}{ Core OPSA-PC5 } \\
\hline OPSA-PC5-45 & Acropora & SUERC-52291 & -1.3 & 340 & 37.7 & Modern & & Modern \\
\hline $\begin{array}{l}\text { OPSA-PC5-95 } \\
\text { OPSA-PC5- }\end{array}$ & Goniastrea & SUERC-52292 & -1.9 & 1051 & 37.7 & 565 & 580 & 613 \\
\hline $\begin{array}{l}140 \\
\text { OPSA-PC5- }\end{array}$ & Montipora & SUERC-52293 & -2.5 & 1137 & 37.7 & 647 & 710 & 680 \\
\hline $\begin{array}{l}210 \\
\text { OPSA-PC5- }\end{array}$ & Euphyllia & SUERC-52294 & -3.4 & 1186 & 37.7 & 674 & 750 & 718 \\
\hline 260 & Lobophyllia & SUERC-52295 & -4.0 & 1384 & 37.7 & 882 & 966 & 921 \\
\hline \multicolumn{9}{|c|}{ Core OPSA-PC6 } \\
\hline $\begin{array}{l}\text { OPSA-PC6-42 } \\
\text { OPSA-PC6- }\end{array}$ & Acropora & SUERC-51880 & -1.3 & 0 & 0 & Modern & & Modern \\
\hline $\begin{array}{l}102 \\
\text { OPSA-PC6- }\end{array}$ & Acropora & SUERC-51881 & -2.0 & 1214 & 37.7 & 692 & 780 & 743 \\
\hline $\begin{array}{l}173 \\
\text { OPSA-PC6- }\end{array}$ & Leptastrea & SUERC-51882 & -3.0 & 1145 & 37.7 & 652 & 716 & 686 \\
\hline 203 & Galaxea & SUERC-51885 & -3.4 & 1190 & 35.7 & 677 & 752 & 720 \\
\hline
\end{tabular}




\begin{tabular}{|c|c|c|c|c|c|c|c|c|}
\hline OPSA-PC7-35 & Acropora & SUERC-52285 & -1.3 & 376 & 35.7 & Modern & & Modern \\
\hline $\begin{array}{l}\text { OPSA-PC7-95 } \\
\text { OPSA-PC7- }\end{array}$ & Goniastrea & SUERC-52286 & -2.1 & 1261 & 37.7 & 738 & 847 & 796 \\
\hline $\begin{array}{l}130 \\
\text { OPSA-PC7- }\end{array}$ & Montipora & SUERC-52287 & -2.6 & 1165 & 37.7 & 662 & 730 & 701 \\
\hline $\begin{array}{l}205 \\
\text { Core OPSA-PC }\end{array}$ & Platygyra & SUERC-52288 & -3.6 & 1207 & 37.7 & 688 & 773 & 736 \\
\hline $\begin{array}{l}\text { OPSA-PC8-35 } \\
\text { OPSA-PC8- }\end{array}$ & Montipora & SUERC-51876 & -1.2 & 0 & 0 & Modern & & Modern \\
\hline $\begin{array}{l}100 \\
\text { OPSA-PC8- }\end{array}$ & Montipora & SUERC-51877 & -2.1 & 686 & 37.7 & 277 & 369 & 328 \\
\hline $\begin{array}{l}140 \\
\text { OPSA-PC8- }\end{array}$ & Turbinaria & SUERC-51878 & -2.7 & 1327 & 37.7 & 815 & 910 & 859 \\
\hline $\begin{array}{l}190 \\
\text { OPSB } \\
\text { Core OPSB-PC }\end{array}$ & Montipora & SUERC-51879 & -3.4 & 1222 & 38.6 & 695 & 789 & 752 \\
\hline OPSB-PC1-36 & Acropora & SUERC-52308 & -1.0 & 386 & 35.7 & Modern & & Modern \\
\hline $\begin{array}{l}\text { OPSB-PC1-84 } \\
\text { OPSB-PC1- }\end{array}$ & Acropora & SUERC-52311 & -1.7 & 1524 & 37.7 & 1011 & 1128 & 1069 \\
\hline $\begin{array}{l}130 \\
\text { OPSB-PC1- }\end{array}$ & Acropora & SUERC-52312 & -2.3 & 2409 & 37.7 & 1979 & 2092 & 2031 \\
\hline $\begin{array}{l}214 \\
\text { Core OPSB-PC }\end{array}$ & Acropora & SUERC-52313 & -3.4 & 1975 & 35.7 & 1471 & 1577 & 1521 \\
\hline OPSB-PC3-50 & Montipora & SUERC-52303 & -0.8 & 513 & 37.7 & Modern & 4 & 126 \\
\hline $\begin{array}{l}\text { OPSB-PC3-80 } \\
\text { OPSB-PC3- }\end{array}$ & Porites & SUERC-52304 & -1.2 & 1557 & 35.7 & 1058 & 1159 & 1107 \\
\hline $\begin{array}{l}150 \\
\text { OPSB-PC3- }\end{array}$ & Acropora & SUERC-52305 & -2.2 & 2261 & 37.7 & 1809 & 1908 & 1857 \\
\hline $\begin{array}{l}197 \\
\text { Core OPSB-PC }\end{array}$ & Montipora & SUERC-52306 & -2.9 & 1857 & 35.7 & 1330 & 1435 & 1388 \\
\hline OPSB-PC4-41 & Montipora & SUERC-52314 & -1.0 & 512 & 37.7 & Modern & 5 & 125 \\
\hline OPSB-PC4-95 & Acropora & SUERC-52315 & -1.8 & 1501 & 35.7 & 975 & 1085 & 1039 \\
\hline
\end{tabular}




\begin{tabular}{|c|c|c|c|c|c|c|c|c|}
\hline $\begin{array}{l}\text { OPSB-PC4- } \\
154 \\
\text { OPSB-PC4- } \\
182\end{array}$ & $\begin{array}{l}\text { Goniastrea } \\
\text { Acropora }\end{array}$ & $\begin{array}{l}\text { SUERC-52316 } \\
\text { SUERC-52317 }\end{array}$ & $\begin{array}{l}-2.6 \\
-3.0\end{array}$ & 2468 & 37.7 & $\begin{array}{l}2033 \\
2019\end{array}$ & 2158 & $\begin{array}{l}2106 \\
2078\end{array}$ \\
\hline \multicolumn{9}{|c|}{ Core OPSB-PC5 } \\
\hline $\begin{array}{l}\text { OPSB-PC5-30 } \\
\text { OPSB-PC5- }\end{array}$ & Acropora & SUERC-52325 & -1.1 & 0 & 0 & Modern & & Modern \\
\hline $\begin{array}{l}143 \\
\text { OPSB-PC5- }\end{array}$ & Acropora & SUERC-52326 & -2.6 & 1815 & 37.7 & 1297 & 1379 & 1345 \\
\hline $\begin{array}{l}200 \\
\text { OPSB-PC5- }\end{array}$ & Porites & SUERC-52327 & -3.3 & 1843 & 35.7 & 1314 & 1410 & 1372 \\
\hline \multicolumn{9}{|c|}{ Core OPSB-PC6 } \\
\hline OPSB-PC6-35 & Acropora & SUERC-52321 & -0.8 & 0 & 0 & Modern & & Modern \\
\hline $\begin{array}{l}\text { OPSB-PC6-70 } \\
\text { OPSB-PC6- }\end{array}$ & Acropora & SUERC-52322 & -1.2 & 1678 & 35.7 & 1192 & 1198 & 1232 \\
\hline $\begin{array}{l}127 \\
\text { OPSB-PC6- }\end{array}$ & Acropora & SUERC-52323 & -2.0 & 1887 & 35.7 & 1372 & 1478 & 1424 \\
\hline $\begin{array}{l}200 \\
\text { OPSC }\end{array}$ & Hydnophora & SUERC-52324 & -3.0 & 1973 & 37.7 & 1465 & 1577 & 1518 \\
\hline \multicolumn{9}{|c|}{ Core OPSC-PC1 } \\
\hline OPSC-PC1-5 & Montipora & SUERC-59749 & -0.5 & 0 & 0 & Modern & & Modern \\
\hline OPSC-PC1-40 & Hydnophora & SUERC-59750 & -1.0 & 790 & 36 & 387 & 475 & 424 \\
\hline $\begin{array}{l}\text { OPSC-PC1-98 } \\
\text { OPSC-PC1- }\end{array}$ & Montipora & SUERC-59751 & -1.8 & 1248 & 36 & 723 & 826 & 779 \\
\hline $\begin{array}{l}140 \\
\text { OPSC-PC1- }\end{array}$ & Porites & SUERC-59752 & -2.4 & 1299 & 38 & 789 & 886 & 833 \\
\hline $\begin{array}{l}182 \\
\text { OPSC-PC1- }\end{array}$ & Montipora & SUERC-59753 & -3.0 & 1286 & 36 & 778 & 877 & 822 \\
\hline $\begin{array}{l}221 \\
\text { OPSC-PC1- }\end{array}$ & Turbinaria & SUERC-59754 & -3.6 & 1508 & 38 & 985 & 1100 & 1047 \\
\hline $\begin{array}{l}268 \\
\text { Core OPSC-PC }\end{array}$ & Galaxea & SUERC-59756 & -4.2 & 1645 & 38 & 1167 & 1250 & 1202 \\
\hline
\end{tabular}




\begin{tabular}{|c|c|c|c|c|c|c|c|c|}
\hline $\begin{array}{l}\text { OPSC-PC2-26 } \\
\text { OPSC-PC2- }\end{array}$ & Montipora & SUERC-59759 & -1.1 & 0 & 0 & Modern & & Modern \\
\hline $\begin{array}{l}103 \\
\text { OPSC-PC2- }\end{array}$ & Montipora & SUERC-59760 & -2.1 & 715 & 38 & 301 & 395 & 352 \\
\hline $\begin{array}{l}170 \\
\text { OPSC-PC2- }\end{array}$ & Euphyllia & SUERC-59761 & -3.0 & 925 & 36 & 491 & 545 & 520 \\
\hline $\begin{array}{l}227 \\
\text { OPSC-PC2- }\end{array}$ & Turbinaria & SUERC-59762 & -3.7 & 1153 & 38 & 656 & 721 & 691 \\
\hline 271 & Turbinaria & SUERC-59763 & -4.3 & 1531 & 36 & 1030 & 1141 & 1077 \\
\hline \multicolumn{9}{|c|}{ Core OPSC-PC3 } \\
\hline $\begin{array}{l}\text { OPSC-PC3-52 } \\
\text { OPSC-PC3- }\end{array}$ & Montipora & SUERC-59764 & -0.8 & 0 & 0 & Modern & & Modern \\
\hline $\begin{array}{l}166 \\
\text { OPSC-PC3- }\end{array}$ & Goniopora & SUERC-59766 & -2.4 & 1104 & 38 & 625 & 686 & 655 \\
\hline $\begin{array}{l}176 \\
\text { OPSC-PC3- }\end{array}$ & Bivalve & SUERC-59769 & -2.5 & 1121 & 36 & 638 & 695 & 667 \\
\hline $\begin{array}{l}190 \\
\text { OPSC-PC3- }\end{array}$ & Hydnophora & SUERC-59770 & -2.7 & 1333 & 38 & 821 & 914 & 864 \\
\hline 222 & Pachyseris & SUERC-59771 & -3.0 & 1557 & 36 & 1058 & 1159 & 1106 \\
\hline \multicolumn{9}{|l|}{ OPSD } \\
\hline \multicolumn{9}{|c|}{ Core OPSD-PC1 } \\
\hline OPSD-PC1-3 & Montipora & SUERC-59772 & -0.1 & 0 & 0 & Modern & & Modern \\
\hline $\begin{array}{l}\text { OPSD-PC1-65 } \\
\text { OPSD-PC1- }\end{array}$ & Goniopora & SUERC-60084 & -0.9 & 1003 & 36 & 544 & 616 & 580 \\
\hline $\begin{array}{l}123 \\
\text { OPSD-PC1- }\end{array}$ & Turbinaria & SUERC-60085 & -1.8 & 1295 & 38 & 785 & 884 & 830 \\
\hline $\begin{array}{l}194 \\
\text { OPSD-PC1- }\end{array}$ & Acropora & SUERC-60087 & -2.8 & 1726 & 38 & 1235 & 1304 & 1271 \\
\hline 240 & Montipora & SUERC-60088 & -3.5 & 1827 & 38 & 1304 & 1392 & 1355 \\
\hline \multicolumn{9}{|c|}{ Core OPSD-PC2 } \\
\hline OPSD-PC2-31 & Acropora & SUERC-60089 & -1.3 & 0 & 0 & Modern & & Modern \\
\hline $\begin{array}{l}\text { OPSD-PC2-72 } \\
\text { OPSD-PC2- }\end{array}$ & Montipora & SUERC-60090 & -1.8 & 997 & 38 & 539 & 613 & 576 \\
\hline 150 & Euphyllia & SUERC-60093 & -2.8 & 2139 & 38 & 1665 & 1785 & 1715 \\
\hline
\end{tabular}




\begin{tabular}{|c|c|c|c|c|c|c|c|c|}
\hline $\begin{array}{l}183 \\
\text { OPSD-PC2- }\end{array}$ & Euphyllia & SUERC-60094 & -3.2 & 2207 & 38 & 1741 & 1849 & 1794 \\
\hline 241 & Turbinaria & SUERC-60095 & -4.0 & 2256 & 38 & 1803 & 1903 & 1851 \\
\hline \multicolumn{9}{|c|}{ Core OPSD-PC3 } \\
\hline OPSD-PC3-21 & Montipora & SUERC-60097 & -1.1 & 0 & 0 & Modern & & Modern \\
\hline $\begin{array}{l}\text { OPSD-PC3-99 } \\
\text { OPSD-PC3- }\end{array}$ & Galaxea & SUERC-60099 & -2.3 & 2066 & 38 & 1573 & 1681 & 1626 \\
\hline $\begin{array}{l}157 \\
\text { OPSD-PC3- }\end{array}$ & Leptoseris & SUERC-60100 & -3.2 & 2358 & 38 & 1911 & 2024 & 1968 \\
\hline 167 & Bivalve & SUERC-60103 & -3.3 & 2421 & 38 & 1991 & 2102 & 2046 \\
\hline
\end{tabular}


Supplementary Table DR2. Mean ( \pm s.d.) relative abundance of coral cover and dominant coral genera across the reefs that form the Paluma Shoals Reef Complex (OPS: Offshore Paluma Shoals, OPSA/B/C/D: Offshore Paluma Shoals A, B, C, D) from Morgan et al., (2016). Classifications of ecological community types at Paluma Shoals North and South can be found in Palmer et al., (2010).

\begin{tabular}{|c|c|c|c|c|c|c|c|c|c|c|}
\hline Benthic cover (\%) & \multicolumn{2}{|c|}{ OPS } & \multicolumn{2}{|c|}{ OPSA } & \multicolumn{2}{|c|}{ OPSB } & \multicolumn{2}{|c|}{ OPSC } & \multicolumn{2}{|c|}{ OPSD } \\
\hline Elevation (m LAT) & 0 & & -0 & & -0. & & -0. & & -0.6 & \\
\hline Live coral & 18.3 & (26) & 22.7 & (31) & 43.9 & (37) & 64.4 & (30) & 54.0 & (36) \\
\hline Acropora spp. & 10.4 & (28) & 30.1 & (41) & 32.1 & (38) & 10.4 & (21) & 25.6 & (33) \\
\hline Montipora spp. & 3.9 & $(18)$ & 7.6 & (23) & 1.2 & $(10)$ & 6.2 & (18) & 21.4 & (30) \\
\hline Turbinaria spp. & 71.3 & (42) & 37.7 & (43) & 62.7 & $(40)$ & 81.3 & (28) & 31.4 & (31) \\
\hline Porites spp. & 2.9 & (15) & 17.0 & (36) & 2.6 & (14) & 0.8 & (6) & 15.7 & (36) \\
\hline Other & 10.7 & $(28)$ & 5.9 & $(22)$ & 1.0 & $(7)$ & 1.4 & $(8)$ & 5.9 & (20) \\
\hline
\end{tabular}




\section{REFERENCES}

Freeman S.P.H.T., Bishop P., Bryant C.L., Cook G.T., Dougans A., Ertunc T., Fallick A., Ganeshram R., Maden C., Naysmith P., Schnabel C., Scott E.M., Summerfield M., Xu S., The SUERC AMS laboratory after 3 years Nuclear Instruments and Methods in Physics Research B 259 (2007) 66-70.

Gillespie, R., 1977, Radiocarbon dating of marine mollusc shells: Australian Quaternary Newsletter v. 9, p. 13-15.

Palmer, S.E., Perry, C.T., Smithers, S.G., and Gulliver, P., 2010, Internal structure and accretionary history of a nearshore, turbid-zone coral reef: Paluma Shoals, central Great Barrier Reef, Australia: Marine Geology, v. 276, no. 1-4, p. 14-29, doi: 10.1016/j.margeo.2010.07.002.

Perry, C.T., Smithers, S.G., and Gulliver, P., 2013, Rapid vertical accretion on a "young" shore-detached turbid zone reef: Offshore Paluma Shoals, central Great Barrier Reef, Australia: Coral Reefs, v. 32, no. 4, p. 1143-1148, doi: 10.1007/s00338-013-1063-8.

Perry, C.T., Smithers, S.G., Gulliver, P., and Browne, N.K., 2012, Evidence of very rapid reef accretion and reef growth under high turbidity and terrigenous sedimentation: Geology, v. 40, no. 8, p. 719-722, doi: 10.1130/G33261.1.

Perry, C.T., Smithers, S.G., Gulliver, P., and Browne, N.K., 2012, Evidence of very rapid reef accretion and reef growth under high turbidity and terrigenous sedimentation: Geology, Supplementary Data, GSA Data Repository 2012199.

Reimer P.J., Bard, E., Bayliss, A., Beck, J.W., Blackwell, P.G., Bronk, Ramsey, C., Buck, C.E., Cheng, H., Edwards, R.L., Friedrich, M., Grootes, P.M., Guilderson, T.P., Haflidason, H., Hajdas, I., Hatt, Ã C., Heaton, T.J., Hogg, A.G., Hughen, K.A., Kaiser, K.F., Kromer, B., Manning, S.W., Niu, M., Reimer, R.W., Richards, D.A., Scott, E.M., Southon, J.R., Turney, C.S.M., van der Plicht, J., 2013, IntCal13 and MARINE13 radiocarbon age calibration curves 0-50000 years calBP Radiocarbon 55(4). DOI: 10.2458/azu_js_rc.55.16947.

Sloata JR, PJ, Jull, TJT, Linick, W and Toolin LJ. 1987, Preparation of small samples for ${ }^{14} \mathrm{C}$ accelerator targets by catalytic reduction of CO. Radiocarbon v. 29(2), p 303-306.

Stuiver, M., and Reimer, P.J., 1993, Extended ${ }^{14} \mathrm{C}$ database and revised CALIB $3.0{ }^{14} \mathrm{C}$ age calibration program. Radiocarbon, v. 35, p. 215-230.

Ulm, S., 2002, Marine and estuarine reservoir effects in Central Queensland, Australia:

Determination of the modern marine calibration curve: Geoarchaeology, v. 17, p. 319348. 